\title{
The Impact of the Coronavirus Disease 2019 (COVID-19) Pandemic on Healthcare Workers with Pre-Existing Psychiatric Conditions
}

\author{
Megan MacKenzie ${ }^{1}$ (D) Sydney Daviskiba ${ }^{1} \cdot$ Miriam Dow $^{1} \cdot$ Peyton Johnston $^{1}$ • \\ Richard Balon $^{2,3} \cdot$ Arash Javanbakht $^{2} \cdot$ Cynthia L. Arfken ${ }^{2}$
}

Accepted: 22 November 2020/ Published online: 7 January 2021

(C) Springer Science+Business Media, LLC, part of Springer Nature 2021

\begin{abstract}
Both healthcare workers (HCWs) and psychiatric patients during the COVID-19 pandemic appear to have elevated prevalence of psychiatric symptoms, but little is known about HCWs with psychiatric diagnoses. To examine their response to the pandemic, we analyzed their perspective, and association with psychiatric symptoms and stress among $\mathrm{HCW}$ with psychiatric diagnosis. Using an online survey of HCW, we analyzed demographics, work information, health factors, open-ended question, sources of stress and standardized mental health scales (Perceived Stress Scale (PSS), Generalized Anxiety Disorder-7 (GAD-7), Patient Health Questionnaire-8 (PHQ-8), and Posttraumatic Stress Disorder Checklist (PCL)). Sixteen out of $129 \mathrm{HCWs}$ reported a pre-existing psychiatric diagnosis (median age 32 years, 8 were females, 8 work in the emergency department). Their perception of the impact severity on symptoms was significantly correlated with all the mental health scales and with stress from avoiding physical contact. In multivariate analysis, PSS score and PCL score were associated with self-rated impact of the COVID19 pandemic on symptoms (standardized beta $=.51$ for PCL and standardized beta $=.55$ for PSS). GAD-7 score was significantly related to both impact (standardized beta $=.44$ ) and stress from avoiding physical contact (standardized beta $=.53$ ). HCWs with psychiatric diagnoses reported a range of perception of the impact of the pandemic on their symptoms with increased severity associated with worse psychiatric outcomes and more stress from avoiding physical contact with others. There is a growing importance to protect HCWs mental health, including those with pre-existing psychiatric diagnosis, and proactively counter psychosocial consequences of healthcare crises.
\end{abstract}

Keywords Stress response $\cdot$ Healthcare workers $\cdot$ COVID-19 $\cdot$ Psychiatry $\cdot$ Stress $\cdot$ Mental health

Megan MacKenzie

megan.mackenzie2@med.wayne.edu

Extended author information available on the last page of the article 


\section{Introduction}

The coronavirus disease 19 (COVID-19) pandemic has both uprooted and impacted lives worldwide, creating a new set of challenges. As of November 10, 2020, global cases have reached over 50.9 million, with over 10 million cases in the United States [1]. The outbreak has resulted in a heavy psychological impact on people, especially those healthcare workers (HCWs) working at the frontline of the pandemic [2]. These unprecedented times are likely to increase severity of pre-existing mental health problems, both during the pandemic and in its aftermath [3]. Our previous study revealed the importance of identifying groups of HCWs at higher-risk of adverse mental health outcomes and the importance of timely wellness interventions [4]. Unfortunately, there is scarce data about the impact of pandemics, such as the current COVID-19 pandemic, on HCWs with preexisting psychiatric diagnoses. Despite messages emphasizing the importance of protecting HCWs' mental health during the current pandemic, few studies have focused on the susceptibility of HCWs with pre-existing psychiatric conditions.

The lesson of timely mental health assessment during the current COVID-19 pandemic was learned from the 2003 severe acute respiratory syndrome (SARS) pandemic, which saw worldwide traumatic stress among HCWs caring for SARS patients [5, 6]. During SARS outbreak, both high-risk (respiratory medicine) and low-risk (non-respiratory medicine) workers in Hong Kong had elevated PSS scores (17 \pm 5.66 , for high-risk, and 15.9 \pm 4.68 for low-risk workers) [6]. However, one year later, stress levels in the high-risk group were much higher $(18.6 \pm 4.9)$ than the low-risk $(14.8 \pm 5.0)$. For comparison, the scores for the high-risk group were higher than the normative value of 12-14 in the US general population, emphasizing the immense psychological impact that healthcare crises can have on more vulnerable HCWs [7]. A similar study conducted during the SARS pandemic on HCWs found that individuals with anxious or avoidant personality traits and avoidant coping mechanisms were at higher risk of mental health stress [8]. Further, problem-focused coping strategies among HCWs during the SARS pandemic has been reported to decrease their stress and lessen their fear [8].

One review of 46 studies across 59 countries and 54,707 participants (mainly frontline nurses and doctors) examined the mental health impact of the COVID-19 pandemic on HCWs [8]. They concluded that HCWs generally reported more anxiety, depression, and sleep problems compared with the general population. The most commonly reported protective factor associated with reduced risk of mental health problems was having social support. However, only a minority of HCWs sought professional help, possibly due to fear of stigma or existing occupational culture [8]. Unfortunately, there is sparse literature on HCWs with previous psychiatric diagnosis and no literature on self-reported impact of the COVID-19 pandemic on their mental health [9]. As people with mental illness or history of mental illness may have different experiences and coping strategies, it is important to examine their perception of the pandemic. To address this concern, we conducted more indepth analysis of our survey of Detroit-area HCWs during the COVID-19 pandemic to assess the sources of stress, perceived level of stress, psychiatric symptoms among HCWs with pre-existing psychiatric diagnoses, and to hear their concerns [4]. This analysis will build upon the higher prevalence of stress and psychiatric symptoms among HCWs with pre-existing psychiatric diagnoses compared to HCWs with neither pre-existing psychiatric diagnoses nor other chronic diseases. 


\section{Methods}

Details of the methods have been reported previously [4]. Briefly, recruitment for this crosssectional study (April 24, 2020 - May 12, 2020) was performed via email distribution (sent out three times) to known Detroit area HCWs and subsequent snowball sampling using Qualtrics platform. Current HCWs in area hospital were eligible. Participants were given information about the study and were asked to complete an anonymous, online survey which included the standardized mental health scales. Completion of the survey implied informed consent. For this analysis we examined HCWs with previous psychiatric diagnosis, demographics, and sources of stressors. Importantly, we asked the HCW with previous psychiatric diagnosis, "Do you feel the current COVID-19 crisis has worsened your psychiatric symptoms?" to see if it had impacted their mental health "not at all", "a little bit", "a moderate amount", or "a great deal".

The standardized mental health scales were the Perceived Stress Scale (PSS), Generalized Anxiety Disorder-7 (GAD-7), Patient Health Questionnaire-8 (PHQ-8), and Posttraumatic Stress Disorder Checklist (PCL) [7, 10-12]. The PSS is the most widely used psychological instrument for measuring perceived stress and has high convergent validity with scales that measure anxiety and depression, as well as inferring clinical consequences [7, 13]. The PSS score ranges from 0 to 40, with scores from 0 to 13 (low), 14-26 (moderate), and 27-40 (severe) perceived stress [7]. The GAD-7 is a valid brief self-report scale for measuring the severity of anxiety, mainly in outpatients [11]. The GAD-7 total score for the seven items ranges from 0 to 21 , with $0-4$ : minimal anxiety, 5-9: mild anxiety, 10-14: moderate anxiety, and 15-21: severe anxiety [11]. The PHQ-8 is a widely used psychological tool with high reliability and validity that measures depressive symptoms [10]. The PHQ-8 total score ranges from 0 to 4: minimal depression, 5-9: mild depression, 10-14: moderate depression, 15-19: moderately severe depression, and 20-24: severe depression [10]. The PCL-5 is a widely used self-report measure of posttraumatic stress symptoms that can be used in a variety of contexts to screen for PTSD symptoms and monitor symptom change during and after treatment [12].

Individual questions were also developed by the researchers. They included self-reported adequacy of personal protective equipment (PPE), resources for patients and training for highly contagious diseases for HCWs. These questions were rated on 7-point Likert scale with anchors of $1=$ extremely inadequate and $7=$ extremely adequate. Individual sources of stress were rated on a 7-point Likert scale with labels of $1=$ Not at all stressful, $4=$ somewhat stressful and $7=$ extremely stressful. These sources of stress included risk of transmitting to family member, friend, and/or member of household, contracting disease, avoiding physical contact with others, finances, not spending time with family/friends, and tension at work.

One open-ended question was included at the end of the survey that stated, "Please include any additional comments regarding your experience with COVID-19 and mental health".

**BLINDED** Institutional Review Board determined that this study was exempt from further review.

\section{Results}

Sixteen persons $(12.4 \%)$ of our total sample of 129 reported a pre-existing psychiatric diagnosis (Table 1). They ranged in age from 27 to 52 (median of 32), 50\% identify as female and one identified as transgender. All were European American, 31.3\% $(n=5)$ lived by 
themselves, 56.3\% ( $n=9)$ were residents, and 50\% worked in the Emergency Department. Two of them had tested positive for COVID and 12 had family/close friend/team member test positive. All of them had someone to provide emotional support, such as talking over problems. Only two had a chronic disease that put them in the higher risk of complications from COVID.

In response to the question on the impact of the pandemic on their symptoms, only one person said, "not at all", six people chose "a little", five people chose "a moderate amount" and four people chose "a great deal". No one chose "a lot". The respondent who responded "not at all"'was a male who had social and emotional support, worked in the Emergency Department, and reported managing stress "moderately well" "during the current crisis; the individual reported finances and avoiding physical contact was "somewhat stressful".

There were five of the 16 respondents who answered the open-ended question at the end of the survey. Below are their responses:

"I feel like I'm going to die, and my program and my institution are completely ok with this. I feel like my death is expected and every day I cry and have nightmares. I've seen ICU fellows walk away and leave me to improvise on codes; I keep having flashbacks to

Table 1 Correlations between self-rated severity of the pandemic on psychiatric symptoms and scores on mental health scales and individual sources of stress

Impact of pandemic $\quad p$ value on symptoms ${ }^{1}$

Work force issues

Adequacy of your training in infectious disease control? ${ }^{2}$

Adequacy of PPE for you? ${ }^{2}$

Adequacy of resources for your patients with COVID? ${ }^{2}$

Number of times your team made decisions regarding allocating life-saving resources?

Number of patients exposed

$\begin{array}{ll}-.16 & .56\end{array}$

$.06 \quad .84$

$.19 \quad .48$

$-.22 \quad .40$

Extent of stress:

Risk of transmitting to family member, and/or member of household $?^{2} \quad .45 \quad .08$

Contracting disease ${ }^{2}$

$.49 \quad .053$

Finance $^{2}$

Not spending times with family/friends ${ }^{3}$

$.13 \quad .63$

Avoiding physical contact with others ${ }^{3}$

Tension at work ${ }^{3}$

Mental health scales

PSS*

GAD-7**

${ }^{1}$ Question was "Do you feel the current COVID-19 crisis has worsened your psychiatric symptoms?" with responses $1=$ not at all to 5 -a great deal

${ }^{2}$ Questions asked, "Please rate the following: adequacy of..." with Likert-type responses of $1=$ Extremely inadequate to $7=$ Extremely adequate

${ }^{3}$ Questions asked, "Please indicate how stress you find the following" with Likert-type responses of $1=$ Not at all stressful, $4=$ Somewhat stressful, and $7=$ Extremely stressful

*Perceived Stress Scale (PSS)

**Generalized Anxiety Disorder Scale (GAD-7)

****Patient Health Questionnaire depression scale (PHQ-8)

****PTSD Checklist for DSM-5 (PCL-5) 
them just saying no because they were infected and the nurses looked to me for what to do. The multitudes of daily horrors not only in the hospital but in the news and from our government. Every day feels worse. I don't want to die but I think that it's gonna happen when I'm finally out of this hell. My soul hurts every day and I have no choice but to keep on working. I hate this. I'm starting to wonder if I made a mistake." (Respondent said the impact on symptoms was "a great deal".)

"I have a history of depression/anxiety and have been managing fine with my daily medications before COVID. Thankfully, I know my coping mechanisms and am seeing a therapist. However, had I not gone through prior depressive episodes; I am unsure if I would have known what to do in this situation" (Respondent said the impact on symptoms was "a great deal".)

"Being furloughed recently has helped my stress regarding the disease but increased my stress regarding the unknown future and finances." (Respondent said the impact on symptoms was "a great deal".)

"Seeing death daily has its effects even when you normally experience death as part of your job. It's also unnerving that hospital systems are inadequately prepared, and your voice goes unheard." (Respondent said the impact on symptoms was "a moderate amount".)

"Resident needs support." (Respondent said the impact on symptoms was "a moderate amount".)

We also examined the correlation between the impact of the pandemic on their symptoms, and the individual specific stressors and mental health scales (Table 1). There was a positive correlation between the increasing severity of the impact on the symptoms and total scores on each of the specific stressors and mental health scales. Additionally, the severity on the impact of the pandemic on their symptoms was positively correlated with stress from avoiding physical contact with others (i.e. social distancing).

To investigate if higher mental health symptoms were associated with more stress of avoiding physical contact or greater severity of impact on symptoms, we conducted regression analysis (with each mental health scale as an outcome) and both impact and stress from avoiding physical contact with others as the independent variables. For the regression model with PCL-5 score as the outcome, the model was significant $(\mathrm{F}(2,12)=6.86, p=.010)$ with only self-reported impact of the pandemic on symptoms (standardized beta $=.51$ ) significant. Similarly, for the regression model with PSS score as the outcome, the model was significant $(\mathrm{F}(2,13)=9.60, p=.003)$ with only self-reported impact of the pandemic on symptoms (standardized beta $=.55$ ) significant. For the regression model with GAD-7 score as the outcome, the model was highly significant $(\mathrm{F}(2,13)=19.164, p<.001)$ with both self-rated impact of the pandemic on symptoms (standardized beta $=.44$ ) and stress from avoiding contact (standardized beta $=.53$ ) significant. For the regression model with PHQ-8 score as the outcome, the model was not significant $(\mathrm{F}(2,13)=3.45, p=.06)$.

\section{Discussion}

To our knowledge, there is no existing literature on COVID-19 pandemic among HCWs with pre-existing psychiatric diagnoses. COVID-19 poses a challenge to all HCWs, but an additional challenge to people with prior psychiatric illness due to disruptions to routines and 
mental health care leading to a potential for relapse or exacerbation of symptoms [14]. To address this important issue, we analyzed a group of frontline HCWs and found that the majority but not all of HCWs with pre-existing psychiatric conditions said the pandemic had affected them and increased their symptoms. Their ratings of the severity of the impact of the pandemic on symptoms was positively correlated with validated measures of stress, anxiety, depression and traumatic stress. Their rating of the severity of the impact of the pandemic on symptoms was also correlated with their rating of stress from avoiding physical contact with others, which may indicate the desire for more human contact.

Some of the HCWs with pre-existing psychiatric diagnoses also wrote about the impact of COVID-19 pandemic. Their words wrote eloquently of previously learned coping mechanisms, but more frequently of the impact of seeing death so often. There was a plea for more support. Understanding HCWs individual perception on the impact that COVID-19 had on their mental health, including feelings of isolation, can help us develop specific strategies to better alleviate their stress [14]. By learning various coping mechanisms or reaching out to mental health professionals before a time of acute stress, HCWs can be better equipped to acknowledge their condition and act on it [15]. Apart from proper training and appropriate PPE for HCWs, access to psychological support and interventions, and ensuring enough rest are important measures to protect their well-being.

Our data add to the emerging data on the psychological impact of COVID-19 on preexisting mental health conditions. One national poll on March 17, 2020, found that 40\% of Americans reported a lifestyle disruption from the COVID-19 pandemic due to social distancing, disruption of services, and unemployment [14]. Respondents who self-identified as having anxiety disorder were concerned about not getting treatment, concern about getting sick and coping poorly [14]. Sixty-four percent of respondents in that study with a mental illness were concerned that their illness would worsen because of the COVID-19 pandemic [14].

Our study was limited by the small size of our convenience sample from one geographic area. Due to the small numbers, we could not analyze the impact of personally contracting COVID-19 or close contact with someone who had contracted the virus. Also, the data collection was past the most acute period during the first wave of infections and during a time of improved supply of PPE and ventilators, and more experience in caring for COVID-19 patients.

In conclusion, our study found psychiatric symptom and severity of stress responses was directly related to their perception of the impact of the COVID-19 pandemic among those frontline HCWs with pre-existing psychiatric conditions. It builds upon our previous report that HCWs with pre-existing psychiatric diagnoses had more stress and psychiatric symptoms than HCWs with neither psychiatric diagnosis nor other chronic diseases [4]. People with pre-existing psychiatric diagnoses are actively working and functioning members of society who may need extra support during this time of enormous stress from the COVID-19 pandemic aggravated by avoidance of physical contact. Unfortunately, mental health stigma persists worldwide, which may limit disclosure of preexisting conditions or discourage seeking out of professional help. To address these barriers, interventions should be pro-active and sensitive.

Acknowledgements The authors would like to express gratitude to Teena Chopra, M.D., Diane Levine, M.D., and Robert Ehrman, M.D., all from Wayne State University School of Medicine, for their invaluable help with distributing the survey. Drs. Chopra, Levine, and Ehrman have no conflicts of interest to declare. 
Authors' Contributions All authors whose names appear on the submission made substantial contributions to the conception, acquisition, analysis, interpretation, and approval of the final version to be published.Data AvailabilityAll data can be made available upon contacting the corresponding author.

\section{Compliance with Ethical Standards}

Conflicts of Interest/Competing Interests The authors declare that there are no competing conflicts of interest.

Ethics Approval The study was approved by the International Review Board at Wayne State University, 87 East Canfield, Second Floor, Detroit, MI 48201. This study was performed in accordance with the ethical standards as laid down in the 1964 Declaration of Helsinki and its later amendments or comparable ethical standards.

Consent to Participate Informed consent was obtained from all individual participants included in the study.

Consent for Publication The participants consented to publication of data collected in this study.

\section{References}

1. Coronavirus Resource Center; COVID-19 Dashboard by the Center for Systems Science and Engineering at John Hopkins University. John Hopkins University \& Medicine. 2020. Available at https://coronavirus.jhu. edu. Accessed September 28, 2020.

2. Bhattacharjee B, Acharya T. The COVID-19 pandemic and its effect on mental health in USA - a review with some coping strategies. Psychiatry Q. 2020;91(4):1135-45. https://doi.org/10.1007/s11126-02009836-0.

3. Esterwood E, Saeed SA. Past Epidemics, Natural Disasters, COVID19, and Mental Health: Learning from History as we Deal with the Present and Prepare for the Future. Psychiatr Q. 2020;91:1121-33. https://doi. org/10.1007/s11126-020-09808-4.

4. Daviskiba S, MacKenzie M, Johnston P, Dow M, Balon R, Javanbakht A, Arfken CL. Rapid assessment of mental health of Detroit-area healthcare workers during the coronavirus disease 2019 pandemic. Annals of Clinical Psychiatry. In Press. September 2020.

5. Maunder RG, Lancee WJ, Balderson KE, Bennett JP, Borgundvaag B, Evans S, et al. Long-term psychological and occupational effects of providing hospital healthcare during SARS outbreak. Emerg Infect Dis. 2006;12(12):1924-32. https://doi.org/10.3201/eid1212.060584.

6. Maunder R, Hunter J, Vincent L, Bennett J, Peladeau N, Leszcz M, et al. The immediate psychological and occupational impact of the 2003 SARS outbreak in a teaching hospital. CMAJ. 2003;168(10):1245-51.

7. Cohen S, Kamarck T, Mermselstein R. A global measure of perceived stress. J Health Soc Behav. 1983;24(4):385-96.

8. Muller AE, Hafstad EV, Himmels JPW, Smedslund G, Flottorp S, Stensland SØ, et al. The mental health impact of the covid-19 pandemic on healthcare workers, and interventions to help them: a rapid systematic review. Psychiatry Res. 2020;293:113441. https://doi.org/10.1016/j.psychres.2020.113441.

9. Asmundson GJG, Paluszek MM, Landry CA, Rachor GS, McKay D, Taylor S. Do pre-existing anxietyrelated and mood disorders differentially impact COVID-19 stress responses and coping? J Anxiety Disord. 2020;74:102271. https://doi.org/10.1016/j.janxdis.2020.102271.

10. Kroenke K, Strine TW, Spitzer RL, Williams JB, Berry JT, Mokdad AH. The PHQ-8 as a measure of current depression in the general population. J Affect Disord. 2009;114(1-3):163-73. https://doi.org/10. 1016/j.jad.2008.06.026.

11. Spitzer RL, Kroenke K, Williams JBW, Löwe B. A brief measure for assessing generalized anxiety disorder: the GAD-7. Arch Intern Med. 2006;166(10):1092-7. https://doi.org/10.1001/archinte.166.10. 1092.

12. Blevins CA, Weathers FW, Davis MT, et al. The Posttraumatic Stress Disorder Checklist for DSM-5 (PCL5): Development and initial psychometric evaluation. Journal of Traumatic Stress. 28:489-98. https://doi. org/10.1002/jts.22059.

13. Babore A, Lombardi L, Viceconti ML, Pignataro S, Marino V, Crudele M, et al. Psychological effects of the COVID-2019 pandemic: perceived stress and coping strategies among healthcare professionals. Psychiatry Res. 2020;293:113366. https://doi.org/10.1016/j.psychres.2020.113366. 
14. Costa M, Pavlo A, Reis G, Ponte K, Davidson L. COVID-19 concerns among persons with mental illness. Psychiatr Serv. 2020 Nov 1;71(11):1188-90. https://doi.org/10.1176/appi.ps.202000245.

15. Tracy D, Tarn M, Eldridge R, Cooke J, Calder J, Greenberg N. What should be done to support the mental health of healthcare staff treating COVID-19 patients? Br J Psychiatry. 2020;217(4):537-9. https://doi.org/ 10.1192/bjp.2020.109.

Publisher's Note Springer Nature remains neutral with regard to jurisdictional claims in published maps and institutional affiliations. 
Megan MacKenzie is a current third-year medical student at Wayne State University School of Medicine who aspires to practice Psychiatry as a future physician. She attended the University of Michigan - College of Literature, Science, and Arts and obtained her B.S. in Microbiology and Minor in International Studies \& Spanish in 2018. Her previous research interests were in stem cell research and regenerative medicine at the University of Michigan and Emergency Medicine research at Wayne State University School of Medicine. She also has an interest in Art Therapy and has an upcoming publication in the AMA Journal of Ethics - July 2021 Issue.

Sydney Daviskiba is a current third-year medical student at Wayne State University School of Medicine who has not yet decided on a field of practice as a future physician. She attended Michigan State University - College of Natural Science, where she graduated with a B.S. in Human Biology and Minors in Economics and Bioethics, Humanities, \& Society (BHS) in 2017. She has prior research experience in Toxicology at Wayne State University School of Medicine.

Miriam Dow is a third-year medical student at Wayne State University School of Medicine. In 2016, she graduated with a B.S. in Biopsychology, Cognition, \& Neuroscience and a minor in Women's Studies from the University of Michigan College of Literature, Science, and the Arts. Due to her interest in Psychiatry she has been focused on developing both research and volunteer initiatives aimed at understanding and addressing community mental health needs.

Peyton Johnston is a current third year medical student at Wayne State University School of Medicine who aspires to practice Obstetrics and Gynecology with a focus in maternal-fetal medicine. She attended Western Michigan University and obtained her Bachelor of Science in Biomedical Sciences and minor in Chemistry and Spanish in 2015. As an undergraduate student, her research interest was in developmental biology, analyzing cell cycle mutations in zebrafish. Currently, Peyton is working on research projects in Emergency Medicine and Obstetrics at Wayne State University School of Medicine.

Richard Balon , M.D. if Professor of Psychiatry and Anesthesiology, Wayne State University School of Medicine, Detroit, Michigan. He is Associate Chair for Education and Faculty Affairs and Program Director of Adul Psychiatry Residency Program at the Department of Psychiatry and Behavioral Neurosciences. His research and academic interests include psychiatric education, human sexuality namely sexual dysfunction associated with psychotropic medications, clinical psychopharmacology and biology of anxiety. He has published widely in these areas, including several books, the last one focusing on Compulsive Sexual Behavior Disorder.

Arash Javanbakht, M.D., is a psychiatrist and serves as the director of the Stress, Trauma, and Anxiety Research Clinic (STARC). His work is focused on anxiety, trauma, and PTSD. He often helps civilians, refugees, and first responders with PTSD. Several research studies at the STARC examine the impact of exposure to war trauma in adults and children Syrian and Iraqi refugees, and biological and psychological factors of risk and resilience. This research examines genetic and inflammation correlates of trauma as well. This work is funded by an NICHD R01 award. Also, use of art, dance and movement, and yoga and mindfulness in helping refugee families overcome stress. STARC also works neurobiology of psychotherapy, and on utilization of augmented reality and telemedicine to develop a method of providing in vivo treatment for anxiety disorders and PTSD. Dr. Javanbakht's work has been featured on the CNN, National Geographic, Aljazeera, NPR, Washington Post, Smithsonian, PBS, American Psychiatric Association Press Briefing, Anxiety and Depression Association of America, American Academy of Child and Adolescent Psychiatry, and tens of other media outlets.

Cynthia L. Arfken , PhD. Dr. Arfken is a Professor in the Department of Psychiatry and Behavioral Neurosciences at Wayne State University in Detroit Michigan. Her training include a PhD in chronic disease epidemiology from Yale University and a postdoctoral fellowship in alcohol research from the University of California in Berkeley. Her research expertise includes mental health services and substance abuse. In addition to research on behavioral health, she is the Vice-Chair of the regional Detroit Wayne Integrated Health Network Substance Use Disorder Oversight Policy Board and one of select epidemiologists reporting use of emerging drugs and drug trends to a national monitoring system. 


\section{Affiliations}

Megan MacKenzie $^{1} \cdot$ Sydney Daviskiba ${ }^{1} \cdot$ Miriam Dow $^{1} \cdot$ Peyton Johnston ${ }^{1} \cdot$ Richard $^{-}$ Balon $^{2,3} \cdot$ Arash Javanbakht $^{2} \cdot$ Cynthia L. Arfken ${ }^{2}$

1 Wayne State University School of Medicine, 4501 Woodward Ave., Apt 511, Detroit, MI 48201, USA

2 Department of Psychiatry and Behavioral Neurosciences, Wayne State University School of Medicine, Detroit, MI, USA

3 Department of Anesthesiology, Wayne State University School of Medicine, MI, Detroit, USA 